\title{
(6) OPEN ACCESS \\ A prospective, randomised, double-masked comparison of local anaesthetic agents for vitrectomy
}

\author{
Ya-li Zhou, ${ }^{1,2}$ Yao Tong, ${ }^{2}$ Yi-xiao Wang, ${ }^{1,2}$ Pei-quan Zhao, ${ }^{2}$ Zhao-yang Wang ${ }^{1,2}$
}

'Department of Ophthalmology, Ninth People's Hospital, Shanghai Jiaotong University School of Medicine, Shanghai, China

${ }^{2}$ Department of Ophthalmology, Xinhua Hospital, Shanghai Jiaotong University School of Medicine, Shanghai, China

\section{Correspondence to}

Dr Zhao-yang Wang, Department of ophthalmology, Ninth People's Hospital, Shanghai Jiao Tong University School of Medicine, 639 Zhizaoju Road, Shanghai 200011, P.R. China; zhaokekewzy@hotmail.com

Received 10 October 2016 Revised 22 November 2016 Accepted 5 December 2016 Published Online First 7 February 2017

\section{ABSTRACT \\ Purpose To compare the intraoperative and postoperative clinical properties of $1 \%$ ropivacaine, $0.75 \%$ bupivacaine, $2 \%$ lidocaine and a mixture of $0.75 \%$ bupivacaine and $2 \%$ lidocaine (bupi+lido) administered for peribulbar anaesthesia during vitrectomy.}

Methods A total of 140 patients were randomly allocated to four groups. The time of onset of analgesia and akinesia was measured. The efficacy of anaesthesia, degree of postoperative pain and intraoperative and postoperative complications were recorded.

Results The mean times of onset $( \pm \mathrm{SD})$ of analgesia for the ropivacaine, bupivacaine, lidocaine and lido+bupi groups were $90.46 \pm 30.08,94.83 \pm 40.72,78.31 \pm 12.56$ and $101.51 \pm 56.94 s$, respectively $(p=0.087)$. The mean times of onset $( \pm S D)$ of akinesia for the ropivacaine, bupivacaine, lidocaine and lido+bupi groups were $138.89 \pm 62.65,151.86 \pm 84.78,122.66 \pm 49.35$ and $141.54 \pm 62.69 \mathrm{~s}$, respectively $(p=0.323)$. No significant difference was observed in the number of patients who attained grade- 5 anaesthesia in the four groups $(p=0.966)$. The outcome of ordered logit analysis showed that the $1 \%$ ropivacaine resulted in a significantly lower degree of postoperative pain compared with the other three groups $(p=0.017$, $p=0.001$ and $p=0.001$, respectively). The incidence of postoperative subconjunctival haemorrhage was decreased in the ropivacaine group compared with the other three groups $(p<0.001)$.

Conclusions For peribulbar anaesthesia in vitrectomy, $1 \%$ ropivacaine alone provides an adequate intraoperative anaesthesia similar to that provided by the bupivacaine, lidocaine and lido+bupi solutions, as well as provides a better quality of postoperative analgesia and decreases postoperative subconjunctival haemorrhage.

Trial registration number ChiCTR-IPR-16007876; Results.

\section{INTRODUCTION}

To accommodate increased patient volumes and simultaneously satisfy economic frugality, local anaesthesia is used more frequently for vitreoretinal surgery. ${ }^{1}$ Moreover, peribulbar block is superior to retrobulbar block due to its higher safety margin. ${ }^{2}{ }^{3}$ For local anaesthetic agents, the most common used in clinical work are ropivacaine, bupivacaine, lidocaine and a mixture of bupivacaine and lidocaine. Among them, ropivacaine has less severe neurotoxicity and cardiotoxicity than bupivacaine. ${ }^{45}$ Bupivacaine has the advantage of a long duration of block, but it is inferior for the onset of anaesthesia when used alone. ${ }^{6}$ Although lidocaine provides a rapid onset of analgesia and akinesia, it has a shorter duration. ${ }^{7}$ So, the mixture with equal volumes of bupivacaine and lidocaine is often used for a quick onset of analgesia and a prolonged duration of action; however, the mixture may lead to a reduction of the advantages of both agents. ${ }^{8}$

Although various agents are used for peribulbar block, there is no consensus regarding the best anaesthetic agent. Some studies have reported that bupivacaine provides a better quality of anaesthesia than a mixture of bupivacaine and lidocaine. ${ }^{9}$ Some have reported that ropivacaine may be a very suitable choice. ${ }^{10}{ }^{11}$ No researchers ever compared the clinical properties of ropivacaine, bupivacaine, lidocaine and a mixture of bupivacaine and lidocaine in one peribulbar anaesthesia study, we therefore conducted this study to compare the intraoperative and postoperative clinical properties of three different agents (1\% ropivacaine, $0.75 \%$ bupivacaine and $2 \%$ lidocaine) and a mixture of $0.75 \%$ bupivacaine and 2\% lidocaine without additional hyaluronidase used for peribulbar block during pars plana vitrectomy.

\section{METHODS}

This prospective, randomised, double-blinded clinical trial was conducted at Xinhua Hospital Affiliated with Shanghai Jiaotong University School of Medicine from December 2015 through July 2016. The study adhered to the tenets of the Declaration of Helsinki. Ethics committee approval was obtained before study initiation. All possible risks and benefits were explained to the eligible patients before enrolment, and informed consent was obtained before any treatment was given. The protocol of this trial has been registered at ChiCTR.org.cn (registration number: ChiCTR-IPR16007876).

\section{Study population}

A total of 140 patients undergoing pars plana vitrectomy for the first time under peribulbar anaesthesia were enrolled in the study. Only one eye from each patient was included. If both eyes of one patient were eligible, the first eye undergoing surgery was included. Eligible participants were adults (aged 18-80 years) who underwent vitrectomy for the first time due to proliferative diabetic retinopathy (PDR), vitreous haemorrhage (VH), retinal detachment (RD), retinal vein occlusion (RVO), macular degeneration (MH) and other diseases (idiopathic macular hole, macular pucker and 
high myopic maculopathy). Communication problems, allergy to amide-type local anaesthetic agents, history of vitreoretinal surgery or orbital surgery, orbital deformity, with episcleral adjunct surgery, trauma, keratitis, conjunctivitis and uveitis were exclusion criteria. There was no patient with posterior synechiae before operation. The diseases that may produce the posterior synechiae were excluded before surgery such as iridocyclitis and Marfan syndrome.

\section{Randomisation and masking}

The random allocation sequence was performed by a statistician according to a computer-generated randomisation list. The random allocation sequence was performed by one of the authors who played no role in the following procedure. The details of the series were unknown to the study investigators and patients until the end of the trial. The patients and investigators were also masked to the type of agent. All anaesthetic solutions were prepared in advance by external personnel that played no further role in this study.

\section{Sample size}

We took into account the time of onset results of a previous study with 30 patients per group. ${ }^{9}$ The aim was to detect a significant difference in the time of onset of anaesthesia among the ropivacaine, bupivacaine, lidocaine and bupi+lido groups. The calculation was based on a 2-tailed test conducted with $\alpha=0.05$ and a power of $80 \% .{ }^{12}$ There was no need to consider potential dropout rate that the follow-up time was 1-2 days after surgery, therefore, the study size was defined as 35 patients per group.

\section{Study procedure}

Eligible patients were allocated randomly into four groups. The patients in the four groups received 1\% ropivacaine (Naropine, AstraZeneca, Sweden), $0.75 \%$ bupivacaine (Sensorcaine, Harvest, Shanghai, China), 2\% lidocaine (Xylocaine, Harvest, Shanghai, China) or a mixture of an equal amount of bupivacaine and lidocaine, respectively, for pars plana vitrectomy, without additional hyaluronidase added to the local anaesthetic solution. ${ }^{13}$

Before surgery, all of the patients were examined and routine laboratory investigations were performed. To prevent rebreathing and ensuing hypercarbia once draped, all of the patients received an oxygen enriched breathing atmosphere. Routine monitoring, including non-invasive arterial blood pressure, heart rate, electrocardiogram, pulse oxygen pulse oximetry and clinical observation were applied before the administration of the peribulbar anaesthesia until the end of the surgery. It is about 5-10 min before the injection, the patients were provided with the appropriate information regarding anaesthesia and surgery, thereby reducing their anxiety. ${ }^{14}$ The peribulbar anaesthesia was always administered by one of the authors who was blinded to the particular anaesthetic and has substantial experience in regional anaesthesia techniques for ophthalmic surgery. Simultaneously, a masked investigator was responsible for scoring the progression of anaesthesia after training.

Each participant received one drop of combination of $0.5 \%$ tropicamide and 5\% phenylephrine eye drops (Mydrin-P, Santen Oy, Japan) in operated eye every $5 \mathrm{~min}$ for four times before the surgery. The patients were asked to look up and not move the eyes. Then, a 25 -gauge steel needle was inserted at the third lateral of the inferior eyelid, with the bevel facing the globe. The steel was directed along the inferior orbital floor to a distance of approximately $25 \mathrm{~mm}, 4-7 \mathrm{~mL}$ of the local anaesthetic agent was injected after gentle negative aspiration for blood.
Then, in the same way, $1-3 \mathrm{~mL}$ was injected at the superior quadrant along the superior orbital roof. The injection was stopped when the globe became tense and firmness in the globe was confirmed by gentle palpation. ${ }^{15}$ After two sites of injection, the globe was massaged with the palm placed over a few pieces of sterile gauze pad using gentle pressure. The total volume of local anaesthetic solution used was recorded. For every $20 \mathrm{~s}$, pressure was released for $5 \mathrm{~s}$ to allow for vascular filling. ${ }^{9}$

Then, the patients were assessed for the efficacy of blockade at $20 \mathrm{~s}$ intervals after the second administration. First, sensory blockade was assessed by touching the cornea with a cotton swab and communication with the patients. Then, the scoring system of Brahma et al ${ }^{16}$ was used for motor blockade. Ocular movement was evaluated in the four quadrants of gaze directions using the following four-point scoring system: 3 (full movement), 2 (moderate movement), 1 (almost no movement) and 0 (akinesia), with a possible total maximum score of 12 points. An ocular movements score of less than 6 and reduced ocular movements in all directions were taken to indicate sufficient block. Once analgesia and akinesia had been achieved, no further assessments were made. The onset time of analgesia and akinesia were defined as the time elapsed from the end of the injection until the best anaesthesia was reached.

If after $5 \mathrm{~min}$ from the time of the end of injection sensory blockade was insufficient and the patients were still feeling pain, a supplementary injection of $1-2 \mathrm{~mL}$ of the test solution was used. If motor blockade was insufficient and the total ocular movement score was 6 or more or there was full movement in one of the four directions, depending on the quadrant, a supplementary injection was administered at the inferior lateral or superior lateral site using 1-2 $\mathrm{mL}$ of the test solution.

During the surgery, 3-port 23-gauge vitrectomy was performed using the Constellation 800 CS System (Alcon Laboratories, Fort Worth, Texas, USA), the patients were encouraged to communicate with the surgeon if pain occurred. If the patients expressed pain, the time of the appearance of pain and what surgical procedures were done at that time were recorded by the investigator. Whether additional anaesthesia was administered depended on the patient.

No injection was given if the patients claimed that the pain was mild and they could endure it. When a patient expressed moderate pain or worse, a supplementary injection was administered as described previously. The duration of the surgical procedure was defined as the time the eye was draped to the time the drape was removed. Complications during surgery were also noted. After surgery, the efficacy of anaesthesia was graded from 0 to 5 and judged by the adequacy of analgesia and akinesia and any supplementary anaesthetic needed to obtain acceptable akinesia. $^{17}$

On the first postoperative day, the degree of pain was recorded using the following five-point verbal rating score: 0 (no pain), 1 (mild pain), 2 (moderate pain), 3 (severe pain) or 4 (unbearable pain). ${ }^{10}$ Any occurrences of subconjunctival haemorrhage, nausea, vomiting, headache, scalp anaesthesia and any untoward event were also recorded. If a patient could not tolerate the postoperative pain, saridon (Compound Paracetamol Tablets, Bayer, China) was given.

\section{Statistical analysis}

Statistical analysis was performed using SPSS software V.22 (SPSS, IBM, Chicago, Illinois, USA). A p value $<0.05$ was considered significantly difference. For continuous variables, the results are presented as the mean $\pm S D$, and the $\mathrm{p}$ values were 
Table 1 Baseline characteristics of the study groups

\begin{tabular}{|c|c|c|c|c|c|}
\hline & $\begin{array}{l}\text { Ropivacaine } \\
\text { group }(n=35)\end{array}$ & $\begin{array}{l}\text { Bupivacaine } \\
\text { group }(n=35)\end{array}$ & $\begin{array}{l}\text { Lidocaine group } \\
(n=35)\end{array}$ & $\begin{array}{l}\text { Lido+bupi group } \\
(n=35)\end{array}$ & p Value \\
\hline Age (years) & $64.31 \pm 9.74$ & $56.46 \pm 11.86$ & $58.54 \pm 13.06$ & $61.86 \pm 12.17$ & 0.03 \\
\hline Sex (male/female) & $15 / 20$ & $19 / 16$ & $18 / 17$ & $14 / 21$ & 0.583 \\
\hline Eye (left/right) & $9 / 26$ & $15 / 20$ & $17 / 18$ & $16 / 19$ & 0.151 \\
\hline Weight $(\mathrm{kg})$ & $67.57 \pm 9.8$ & $69.27 \pm 8.26$ & $65.97 \pm 9.8$ & $65.19 \pm 13.96$ & 0.391 \\
\hline Type of disease (PDR/RRD/macular diseases/RVO/others) & $3 / 18 / 11 / 1 / 2$ & $5 / 18 / 9 / 0 / 3$ & $10 / 11 / 9 / 1 / 4$ & $7 / 13 / 12 / 2 / 1$ & 0.489 \\
\hline Patients with high myopia ( $\geq 6 \mathrm{D}), \mathrm{n}$ & 7 & 4 & 5 & 4 & 0.706 \\
\hline Patients who underwent phaco-ppv surgery, $n$ & 31 & 29 & 26 & 33 & 0.112 \\
\hline Duration of surgery (min) & $86.06 \pm 26.09$ & $81.66 \pm 23.49$ & $78.49 \pm 27.55$ & $87.17 \pm 24.6$ & 0.458 \\
\hline
\end{tabular}

PDR, proliferative diabetic retinopathy; ppv, pars plana vitrectomy; RRD, rhegmatogenous retinal detachment; RVO, retinal vein occlusion.

Table 2 Characteristics of anaesthesia in four groups

\begin{tabular}{|c|c|c|c|c|c|}
\hline & Ropivacaine group $(n=35)$ & Bupivacaine group $(n=35)$ & Lidocaine group $(n=35)$ & Lido+bupi group ( $n=35)$ & $p$ Value \\
\hline Volume of anaesthetic (mL) & $7.69 \pm 0.98$ & $7.4 \pm 0.99$ & $7.63 \pm 0.95$ & $7.59 \pm 1.03$ & 0.695 \\
\hline Time of onset of analgesia (s) & $90.46 \pm 30.08$ & $94.83 \pm 40.72$ & $78.31 \pm 12.56$ & $101.51 \pm 56.94$ & 0.087 \\
\hline Time of onset of akinesia (s) & $138.89 \pm 62.65$ & $151.86 \pm 84.78$ & $122.66 \pm 49.35$ & $141.54 \pm 62.69$ & 0.323 \\
\hline Patients expressed pain during surgery, $n$ & 0 & 4 & 5 & 5 & 0.931 \\
\hline Patients attained grade- 5 anaesthesia, $n$ & 35 & 34 & 34 & 31 & 0.966 \\
\hline
\end{tabular}

obtained by one-way analysis of variance between the groups. For categorical variables, the results are presented as the frequency and the percentage, and the $\mathrm{p}$ values were obtained by Pearson's $\chi^{2}$ test. The outcome of the postoperative pain degrees was analysed using ordered logit analysis adjusted for age at baseline.

\section{RESULTS}

Patient characteristics, types of diseases, patients with high myopia $(\geq 6 \mathrm{D})$, patients who underwent phaco-pars plana vitrectomy surgery and duration of surgery were similar among the four study groups except for age (table 1). Table 2 shows the volumes of anaesthetic, time of onset of sensory and motor blockade, the number of patients who expressed pain or attained a grade-5 block during surgery. The numbers of patients who experienced pain during surgery were the following: no patients in the ropivacaine group $(0 \%)$, four in the bupivacaine group (11.4\%), five in the lidocaine group (14.3\%) and five in the lido+bupi group $(14.3 \% ; p=0.931)$. No significant difference was observed for the number of patients who attained grade-5 anaesthesia in the ropivacaine group $(n=35 ; 100 \%)$, bupivacaine group $(n=34 ; 97.1 \%)$, lidocaine group $(n=34$; 97.1\%) and lido+bupi group $(n=31 ; 88.6 \% ; p=0.966)$.

During the first day after surgery, no patient in any group experienced unbearable pain. The number of patients and corresponding degree of pain are shown in figure 1 . The outcome of ordered logit analysis showed that age and anaesthetic agents were associated with postoperative pain and were two independent protective factors of postoperative pain (table 3). For age, there was a negative correlation between age and postoperative pain that the young patients were more likely to experience a higher degree of pain than the older patients $(p<0.001)$. For anaesthetic agents, $1 \%$ ropivacaine resulted in a significantly lower degree of pain compared with the other three groups $(\mathrm{p}=0.017, \mathrm{p}=0.001$ and $\mathrm{p}=0.001$, respectively), while no significant difference among the other three groups was found.

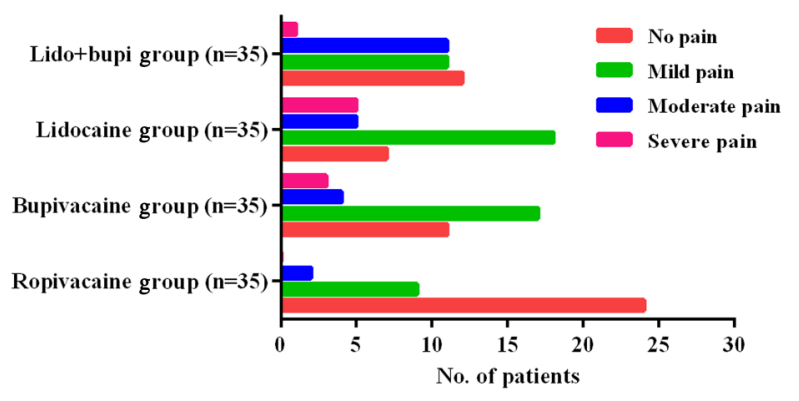

Figure 1 Bar graph showing the number of patients who experienced pain during the first postoperative day using verbal rating scale score of pain.

No adverse events were noted during the delivery of four types of anaesthesia during surgery. The incidence of subconjunctival haemorrhage after surgery was significantly lower in the ropivacaine group compared with the bupivacaine, lidocaine and lido+bupi groups $(\mathrm{p}=0.001)$. Adverse events including nausea, vomiting, headache, dizziness and scalp anaesthesia were not significantly different among the four groups (figure 2).

\section{DISCUSSION}

A quick onset of anaesthesia with prolonged intraoperative analgesia and better postoperative comfort is a desired goal in local anaesthesia for ophthalmic surgery. Ropivacaine and bupivacaine are both long-acting, amide-type local anaesthetics. Ropivacaine is marketed as a pure $\mathrm{S}(-)$-enantiomer, whereas bupivacaine is a racemate. Lidocaine is a short-acting amide-type local anaesthetic. The proton binding affinity $(\mathrm{pKa})$ values determine the penetration time of the solution, and the specific $\mathrm{pKa}$ values are 7.7 for lidocaine and 8.1 for both ropivacaine and bupivacaine, which largely determine the onset of local analgesia. ${ }^{18}$ Agents with lower $\mathrm{pKa}$ constants provide a more rapid analgesic onset. The plasma binding rate of protein is $94 \%, 95 \%$ and $64 \%$ for bupivacaine, ropivacaine and lidocaine, respectively. ${ }^{9} 17$ The 
Table 3 Outcome of total analysis using ordered logit about postoperative pain

\begin{tabular}{|c|c|c|c|c|c|c|}
\hline & \multicolumn{6}{|c|}{ Ordered logit analysis $(n=140)$} \\
\hline & Estimate & p Value & Estimate & p Value & Estimate & p Value \\
\hline \multicolumn{7}{|l|}{ Threshold } \\
\hline No pain & -8.582 & 0.001 & -8.767 & 0.001 & -8.139 & 0.001 \\
\hline Mild pain & -6.399 & 0.013 & -6.583 & 0.009 & -5.955 & 0.017 \\
\hline Moderate pain & -4.742 & 0.063 & -4.926 & 0.048 & -4.298 & 0.083 \\
\hline \multicolumn{7}{|l|}{ Intercept } \\
\hline Age & -0.058 & $0.000 *$ & -0.058 & $0.000 *$ & -0.058 & $0.000 *$ \\
\hline Weight & -0.028 & 0.175 & -0.028 & 0.175 & -0.028 & 0.175 \\
\hline Duration of surgery & 0.008 & 0.275 & 0.008 & 0.275 & 0.008 & 0.275 \\
\hline Volume of anaesthetic & -0.278 & 0.175 & -0.278 & 0.175 & -0.278 & 0.175 \\
\hline \multicolumn{7}{|l|}{ Sex } \\
\hline Female & -0.453 & 0.327 & -0.453 & 0.327 & -0.453 & 0.327 \\
\hline Male & 0 & - & 0 & - & 0 & - \\
\hline \multicolumn{7}{|l|}{ Eye } \\
\hline Left & -0.464 & 0.194 & -0.464 & 0.194 & -0.464 & 0.194 \\
\hline Right & 0 & - & 0 & - & 0 & - \\
\hline PDR & 0.363 & 0.635 & 0.363 & 0.635 & 0.363 & 0.635 \\
\hline RRD & -0.542 & 0.469 & -0.542 & 0.469 & -0.542 & 0.469 \\
\hline \multicolumn{7}{|l|}{ Type of disease } \\
\hline Macular diseases & -0.209 & 0.769 & -0.209 & 0.769 & -0.209 & 0.769 \\
\hline RVO & -0.783 & 0.531 & -0.783 & 0.531 & -0.783 & 0.531 \\
\hline Others & 0 & - & 0 & - & 0 & - \\
\hline Ropivacaine group $(n=35)$ & -1.698 & $0.001 *$ & -1.883 & $0.001^{*}$ & -1.255 & $0.017^{\star}$ \\
\hline Bupivacaine group $(n=35)$ & -0.443 & 0.355 & -0.628 & 0.188 & 0 & - \\
\hline Lidocaine group $(n=35)$ & 0.184 & 0.639 & 0 & - & 0.628 & 0.188 \\
\hline Lido+bupi group $(n=35)$ & 0 & - & -0.184 & 0.693 & 0.443 & 0.355 \\
\hline
\end{tabular}

Figure 2 Bar graph showing the number of patients with adverse reactions after surgery.

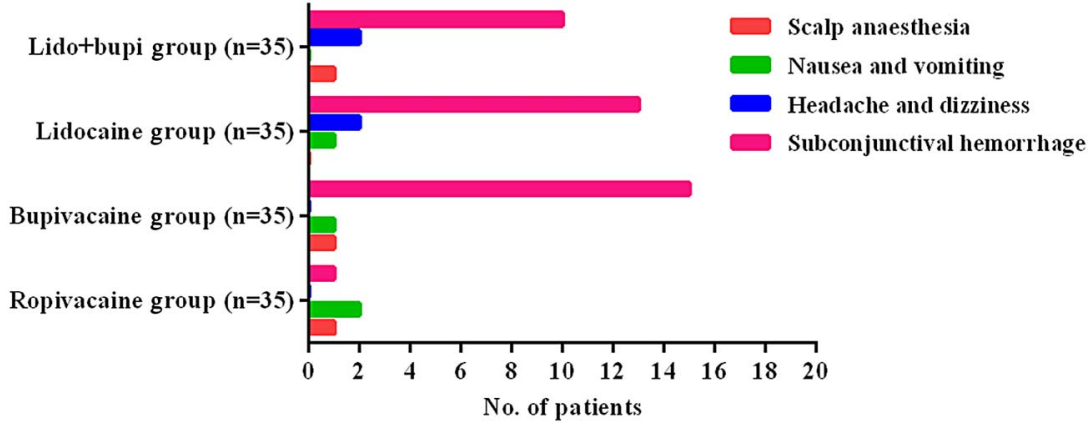

more capable an anaesthetic binds to protein, the longer the duration of action. The mixture of bupivacaine and lidocaine is often used based on the theoretical belief that this mixture provides a quicker onset and a longer duration of analgesia.

In our study, there was no significant difference in the time of onset of both analgesia $(p=0.087)$ and akinesia $(p=0.323)$ among the four groups. The findings are similar to the results of those of Jaichandran et $a l^{9}$ and Gioia et $a l,{ }^{10}$ who conducted a study on peribulbar anaesthesia for vitreoretinal surgery.

No significant difference was observed in the number of patients who experienced any pain during surgery in the ropivacaine $(n=0)$, bupivacaine $(n=4)$, lidocaine $(n=5)$ and lido+bupi groups $(n=5 ; p=0.931)$. Among them, only one patient in the bupivacaine group (25\%), one in the lidocaine group (20\%) and three in the lido+bupi group (80\%) required a one-time supplementary block. Additionally, most patients attained a grade-5 block and there was no significant difference among the four groups $(p=0.966)$ likely due to the short duration of the surgery. ${ }^{9}$ Contrary to our results, a study conducted by Jaichandran et al stated that the 2\% lidocaine and the mixture solution caused significantly more patients to experience pain than the $0.75 \%$ bupivacaine during surgery, and all of the patients required a supplementary block at least once, and some patients were even supplemented twice. This difference may be due to the shorter duration of surgery. In our study, the mean duration times of surgery were approximately $80 \mathrm{~min}$ in the four groups. In the study by Jaichandran et al, the duration times were approximately $180 \mathrm{~min}$ and they concluded that bupivacaine was a better choice for local anaesthetic solution. Obviously, the duration of surgery in our study was far less than that conducted by Jaichandran et al, short duration of the surgery may be the main reason for only few patients required 
supplementary block and most patients attained satisfactory anaesthetic effect in our study. Ropivacaine is a long-acting local anaesthetic, which shows an identical efficacy to bupivacaine, with similar analgesic duration over hours when used alone. ${ }^{18}$ Therefore, we firmly believe that ropivacaine is able to sustain analgesia during a longer operation time in peribulbar block. Moreover, ropivacaine has less severe neurotoxicity and cardiotoxicity than bupivacaine. ${ }^{45}$ Long duration and less toxicity of ropivacaine make it a better choice than bupivacaine in retina surgery.

In our study, we found that ropivacaine can provide effective postoperative pain relief and was superior to the other three agents. When the age of patients in our study is not consistent among the four groups, we compared risk-adjusted outcomes using ordered logit analysis that adjusted for patient factors, which included age, weight, duration of surgery, volume of anaesthetic, sex (male/female), eye (left/right), five types of diseases and four anaesthetic groups. Diseases included PDR, rhegmatogenous RD (RRD), macular diseases, RVO and others. The outcome of ordered logit analysis showed that either age or anaesthetic agents were associated with postoperative pain, and they were two independent protective factors for postoperative pain (table 3). No other factors had an effect on the degree of postoperative pain. Our findings regarding age, which was negatively correlated with postoperative pain that the young patients were more likely to experience a higher degree of pain than the older patients $(\mathrm{p}<0.001)$, was consistent with the conclusion of a previous study. ${ }^{19}$ For anaesthetic agents, $1 \%$ ropivacaine resulted in a significantly lower degree of pain compared with the other three groups $(p=0.017, p=0.001$ and $p=0.001$, respectively), which was superior to the other three agents, while no significant difference was observed among the other three groups. From our study, we concluded that ropivacaine can provide effective pain relief during the postoperative period, which is consistent with the conclusions of previous studies. ${ }^{10} 18$

Subconjunctival haemorrhage subsided within 3-7 days after surgery; however, the incidence was significantly lower in the ropivacaine group compared with the bupivacaine group, lidocaine group and lido+bupi group (figure $2 ; \mathrm{p}=0.001$ ). The low incidence of subconjunctival haemorrhage could be explained by the intrinsic vasoconstrictive properties of ropivacaine. ${ }^{20} 21$ In addition, all included patients required sutures during vitrectomy, the cases that had subconjunctival haemorrhage had a relatively more uncomfortable feeling at the incision because of knot friction sensitivity. So, ropivacaine is effective in reducing subconjunctival haemorrhage and providing more comfortable feeling than the other three solutions in vitrectomy.

This study has several limitations. One limitation includes the inconsistent baseline for age. The eligible participants were the adults in 18-80 age range, and the age gap was great. Only $17.9 \%$ of patients in the study were in the $18-49$ age range $(\mathrm{n}=25 ; 17.9 \%)$, the rest of the patients were aged 50 years or older. The outcome of ordered logit analysis showed that age and anaesthetic agents were two independent protective risk factors of postoperative pain. For age, there was a negative correlation between age and postoperative pain that the young patients were more likely to experience a higher degree of pain than the older patients $(p<0.001)$. So, the age range of the eligible patients should be limited in the future study. Additionally, a mixture of ropivacaine and bupivacaine and a mixture of ropivacaine and lidocaine groups could be included in future studies, but the mixture of ropivacaine and lidocaine or ropivacaine and bupivacaine are seldom used in eye surgery anaesthesia in clinical practice. Ropivacaine and bupivacaine have identical efficacy and potency, with similar analgesic duration, and thus their mixture may not be necessary. ${ }^{18}$ Only a few studies have considered a ropivacaine and lidocaine mixture in eye surgery, and the safety of the mixture used in peribulbar anaesthesia is unclear. For all patients, we covered the operated eye with a sterile gauze pad and iron goggle for at least 6 hours postoperatively. The exact time of termination of the motor blockade of all the patients was also not measured.

\section{CONCLUSIONS}

Despite these limitations, this study suggests that $1 \%$ ropivacaine alone is a suitable choice when administering peribulbar anaesthesia for patients undergoing pars plana vitrectomy because it produces an adequate quality of intraoperative anaesthesia and better postoperative anaesthesia and also improves patient comfort compared with bupivacaine, lidocaine and the mixture of lidocaine and bupivacaine.

Contributors Z-yW contributed to the design, interpretation, surgery procedure and made revisions to the manuscript. Y-IZ contributed to experimental data acquisition and wrote the first draft. YT contributed to online registration and data acquisition. Y-xW and P-qZ contributed to the data analysis.

Funding This work was supported by Project of the National Natural Science Funds of China (No. 81371040) and Shanghai Pujiang Program (No. 15PJD028).

Competing interests None declared.

Patient consent Obtained.

Ethics approval The Ethics Committee of Xinhua Hospital affiliated with Shanghai Jiaotong University School of Medicine.

Provenance and peer review Not commissioned; externally peer reviewed.

Open Access This is an Open Access article distributed in accordance with the Creative Commons Attribution Non Commercial (CC BY-NC 4.0) license, which permits others to distribute, remix, adapt, build upon this work non-commercially, and license their derivative works on different terms, provided the original work is properly cited and the use is non-commercial. See: http://creativecommons.org/ licenses/by-nc/4.0/

\section{REFERENCES}

1 Newsom RS, Wainwright AC, Canning CR. Local anaesthesia for 1221 vitreoretinal procedures. Br J Ophthalmol 2001;85:225-7.

2 Weiss JL, Deichman CB. A comparison of retrobulbar and periocular anesthesia for cataract surgery. Arch Ophthalmol 1989;107:96-8.

3 Demediuk OM, Dhaliwal RS, Papworth DP, et al. A comparison of retrobulbar and periocular anesthesia for vitreoretinal surgical procedures. Arch Ophthalmol 1995; 113:908-13.

4 Knudsen K, Beckman Suurküla M, Blomberg S, et al. Central nervous and cardiovascular effects of i.v infusions of ropivacaine, bupivacaine and placebo in volunteers. Br J Anaesth 1997;78:507-14.

5 Scott DB, Lee A, Fagan D, et al. Acute toxicity of ropivacaine compared with that of bupivacaine. Anesth Analg 1989;69:563-9.

6 Zand F, Razavizadeh MR, Azemati S. Comparative study of onset and duration of action of $0.5 \%$ bupivacaine and a mixture of $0.5 \%$ bupivacaine and $2 \%$ lidocaine for epidural anaesthesia. Acta Medica Iranica 2004;42:256-8.

7 Oji E, Oji A. Bupivacaine and lignocaine for ophthalmic surgery. Br J Ophthalmol 1987;71:66-8.

8 Chin GN, Almquist HT. Bupivacaine and lidocaine retrobulbar anaesthsia. A double-blind clinical study. Ophthalmology 1983;90:369-72.

9 Jaichandran VV, Raman R, Gella L, et al. Local anesthetic agents for vitreoretinal surgery: no advantage to mixing solutions. Ophthalmology 2015;122:1030-3.

10 Gioia L, Prandi E, Codenotti $M$, et al. Peribulbar anesthesia with either $0.75 \%$ ropivacaine or a $2 \%$ lidocaine and $0.5 \%$ bupivacaine mixture for vitreoretinal surgery: a double-blinded study. Anesth Analg 1999;89:739-42.

11 Seidenari P, Santin G, Milani P, et al. Peribulbar and retrobulbar combined anesthesia for vitreoretinal surgery using ropivacaine. Eur J Ophthalmol 2006;16:295-9.

12 Browner WS, Black D, Newman B, et al. Estimating sample size and power In: Hulley SB, Cummings SR, eds. Designing clinical research-an epidemiologic approach. Baltimore: Williams \& Wilkins, 1988:139-50.

13 Gouws $P$, Galloway $P$, Jacob J, et al. Comparison of articaine and bupivacaine/ lidocaine for sub-Tenon's anaesthesia in cataract extraction. $\mathrm{Br} J$ Anaesth 2004;92:228-30. 
14 Kumar CM, Eke T, Dodds C, et al. Local anaesthesia for ophthalmic surgery-new guidelines from the Royal College of Anaesthetists and the Royal College of Ophthalmologists. Eye (Lond) 2012;26:897-8.

15 Bloomberg LB. Administration of periocular anesthesia. J Cataract Refract Surg 1986;12:677-9.

16 Brahma AK, Pemberton CJ, Ayeko M, et al. Single medial injection peribulbar anaesthesia using prilocaine. Anaesthesia 1994;49:1003-5.

17 Sharma T, Gopal L, Parikh S, et al. Parabulbar anaesthesia for primary vitreoretinal surgery. Ophthalmology 1997;104:425-8.
18 Owen MD, Dean LS. Ropivacaine. Expert Opin Pharmacother 2000;1:325-36.

19 Omulecki W, Laudanska-Olszewska I, Synder A. Factors affecting patient cooperation and level of pain perception during phacoemulsification in topical and intracameral anesthesia. Eur J Ophthalmol 2009:19: 977-83.

20 Zink W, Graf BM. Benefit-risk assessment of ropivacaine in the management of postoperative pain. Drug Saf 2004;27:1093-114.

21 Govêia CS, Magalhães E. Ropivacaine in peribulbar anesthesia-vasoconstrictive properties. Rev Bras Anestesiol 2010;60:495-512. 